\title{
Higher level of faecal SCFA in women correlates with metabolic syndrome risk factors
}

\author{
Tatiana F. S. Teixeira ${ }^{1 *}$, Łukasz Grześkowiak ${ }^{2}$, Sylvia C. C. Franceschini ${ }^{1}$, Josefina Bressan ${ }^{1}$, \\ Célia L. L. F. Ferreira ${ }^{3}$ and Maria C. G. Peluzio ${ }^{1}$ \\ ${ }^{1}$ Department of Nutrition and Health, University Campus, Viçosa, Minas Gerais 36570-000, Brazil \\ ${ }^{2}$ Functional Foods Forum, University of Turku, Turku, Finland \\ ${ }^{3}$ Department of Food Technology, University Campus, Viçosa, Minas Gerais 36570-000, Brazil
}

(Submitted 11 October 2011 - Final revision received 19 March 2012 - Accepted 14 May 2012 - First published online 4 July 2012)

\begin{abstract}
SCFA provide energy to the host and influence lipid and glucose metabolism, suggesting that they may have an impact on the occurrence of metabolic risk factors. The aim of the present study was to determine the concentration of SCFA in faeces of lean and obese individuals and to analyse whether associations between faecal SCFA and metabolic syndrome parameters are present. Lean $(n 20)$ and obese $(n 20)$ women of similar age (28.5 (SD 7.6) v. 30.7 (SD 6.5) years, $P=0.33$ ) participated in the study. Anthropometric measurements, body composition, blood pressure and biochemical parameters were assessed. SCFA were extracted from faeces and quantified by GC. Blood pressure and blood glucose, although within the normal limits, were higher in the obese group compared to lean subjects $(P<0 \cdot 05)$. Lower HDL concentration and higher insulin and homeostasis model assessment (HOMA) index were observed in the obese than in the lean group $(P<0 \cdot 05)$. The median values of SCFA $(\% \mathrm{w} / \mathrm{w})$ from the lean and obese groups were butyric $(0 \cdot 021 v \cdot 0 \cdot 044, P=0 \cdot 024)$, propionic $(0.021 v \cdot 0.051, P=0.007)$ and acetic $(0.03 v \cdot 0.061, P=0.01)$. SCFA correlated positively with metabolic syndrome risk factors such as adiposity, waist circumference and HOMA index $(P<0.05)$, and inversely with HDL $(P<0 \cdot 05)$. Our results suggest that the higher faecal concentration of SCFA is associated with metabolic risk factors and thus may influence metabolic homeostasis.
\end{abstract}

Key words: SCFA: Obesity: Insulin: Homeostasis model assessment: HDL

The definition and criteria for the diagnosis of the metabolic syndrome have been discussed intensively worldwide. However, the concept of the term 'metabolic syndrome' is well known and relates to the clustering of the following metabolic risk factors: impaired glucose tolerance, high blood pressure, elevated TAG levels, low HDL-cholesterol levels, insulin resistance and central adiposity. The last two factors have been considered to contribute to the occurrence of all the others $^{(1,2)}$. The 'metabolic syndrome' concept reminds health professionals about the importance of assessing each related risk factor. This would allow an early detection of risk factors and the establishment of strategies for the reduction of incidence of CVD and diabetes.

It is well recognised that an excessive supply of energy to the host associated with low expenditure plays an important role in the development of obesity ${ }^{(3)}$. The enlargement of fat depots, particularly abdominal obesity, favours the development of insulin resistance ${ }^{(4)}$. The gastrointestinal tract is the site of absorption of nutrients that provide energy to the host. The interactions between dietary molecules, physiological conditions (digestive fluids, hormones secretion, motility, absorption rate) and microbiota within the gastrointestinal tract influence metabolic homeostasis ${ }^{(5)}$.

The gut microbiota has been suggested to be involved in the control of body weight and regulation of insulin resistance $^{(6,7)}$. The mechanisms proposed by which the microbiota would interact with the metabolism of the host include influence on gut-hormone production, intestinal permeability and endotoxaemia, and the provision of additional energy through SCFA ${ }^{(8,9)}$.

SCFA are organic fatty acids with one to six carbons. The most important SCFA for human metabolism are acetic, propionic and butyric, which arise mainly from bacterial fermentation in the gut ${ }^{(10)}$. They can provide energy and are possibly involved in lipid and glucose metabolism in the host ${ }^{(10)}$, suggesting that they may have an impact on the occurrence of metabolic risk factors.

Abbreviation: HOMA, homeostasis model assessment.

*Corresponding author: T. F. S. Teixeira, fax +55 313899 3176, email tatifichee@hotmail.com 
In fact, an increased concentration of SCFA in faeces has been related to higher body weight gain in an animal model $^{(11)}$ and in obese humans ${ }^{(12)}$. In both studies, differences in gut microbiota composition were also reported. This has led to the hypothesis that differences in gut microbiota composition between the lean and the obese might contribute to a higher capacity to harvest energy from the diet by an increased production of SCFA, subsequently leading to weight imbalance. However, associations between faecal SCFA with different metabolic risk factors have not yet been reported. Hence, the aim of the present study was to determine the concentration of SCFA in faeces of lean and obese women and analyse whether associations between SCFA and variables included in the concept of the metabolic syndrome, such as abdominal obesity, glucose, insulin, TAG and HDL levels, are present.

\section{Experimental methods \\ Subjects}

The recruitment of female volunteers occurred using written announcements. Women interested in participating in the study were interviewed by phone. The subjects were selected based on the following criteria: age over 18 years, not pregnant or breast-feeding, free of any liver, thyroid or gastrointestinal disease, not taking any kind of supplements or medications in the last 6 months, except oral contraceptives, being lean (BMI $18.5-24.9 \mathrm{~kg} / \mathrm{m}^{2}$ ) or obese (BMI $\left.>30 \mathrm{~kg} / \mathrm{m}^{2}\right)$. The subjects were taken into the study after they provided a written informed consent. This study was conducted according to the guidelines laid down in the Declaration of Helsinki and all procedures involving human subjects were approved by the Ethical Committee of the Federal University of Viçosa (protocol number 001/2010, Federal University of Viçosa).

A total of twenty lean females (BMI 21.5 (sD 1.39) kg/m²) and twenty obese females (BMI $35.04(\mathrm{SD} 3.98) \mathrm{kg} / \mathrm{m}^{2}$ ) of similar age (mean age of the lean and obese groups $28 \cdot 5$ (SD 7.6) $v$. 30.7 (sD 6.5 ),$P=0.33$ ) participated in the study. According to a recent brief medical history, all subjects were healthy.

The subjects were evaluated at the Laboratory of Energetic Metabolism and Body Composition. After fasting for $10 \mathrm{~h}$, they were weighed (wearing light clothes). Their body composition was analysed by tetra polar bio-impedance (BodySystems $\left.{ }^{\circledR}\right)$. Blood pressure was measured and blood samples were collected for biochemical analyses. Fresh faecal samples were collected and then immediately frozen at $-20^{\circ} \mathrm{C}$.

\section{Biochemical analysis}

Blood samples were analysed at the Laboratory of Clinical Analysis of the Health Division at the Federal University of Viçosa. The biochemical assessments included total cholesterol and lipoproteins (enzymatic colorimetric method), aspartate and alanine aminotransferases (kinetic colorimetric method), fasting plasma glucose (enzymatic colorimetric method of glucose-oxidase) (all the kits used were purchased from Bioclin/Quibasa) and insulin through the electrochemiluminescence method using the Modular Analytics E170 e Elecsys
2010 (Roche Diagnostics ${ }^{\circledR}$ ). LDL concentration was estimated by the Friedwald formula ${ }^{(13)}$. The homeostasis model assessment (HOMA) index was used as an indicator of insulin resistance and calculated as follows ${ }^{(14)}$ :

$$
\begin{aligned}
\text { HOMA }= & \text { fasting glucose }(\mathrm{mmol} / \mathrm{l}) \\
& \times \text { fasting insulin }(\mathrm{mU} / \mathrm{l}) / 22 \cdot 5 .
\end{aligned}
$$

Insulin resistance was diagnosed based on HOMA $>2 \cdot 71$ according to Geloneze et al. ${ }^{(15)}$.

\section{Food intake}

Volunteers were instructed to fill in three food records on nonconsecutive days and including one weekend day. Food records were analysed using the software DietWin Professional ${ }^{\circledR}$, and the mean value from the $3 \mathrm{~d}$ was used for subsequent statistical comparison of the main macronutrients and total fibre intake between the groups and its correlation with SCFA concentration.

\section{Faecal SCFA analysis}

The extraction of SCFA was based on the method of SmirickyTjardes et al. ${ }^{(16)}$. Briefly, around $800 \mathrm{mg}$ of frozen faeces were weighed and homogenised with the addition of $1 \mathrm{ml}$ of $m$-phosphoric acid solution (25\%). For each sample, this step was performed in duplicate. After incubation at room temperature for $30 \mathrm{~min}$, the samples were centrifuged (Refrigerated microcentrifuge, HERMLE Z 216MK; Hermle Labortechnik) at $17319 \mathrm{~g}$ for $30 \mathrm{~min}$ at $4^{\circ} \mathrm{C}$. Then, the supernatants were transferred to a new Ependorf tube. After a second centrifugation, the supernatants were collected and subsequently frozen at $-20^{\circ} \mathrm{C}$. Before analysis, a third centrifugation and supernatant collection were performed. The final volume of supernatants from each duplicate was mixed together and homogenised. Butyric, propionic and acetic acids were measured by GC (model CG-17A; Shimadzu ${ }^{\circledR}$ ) equipped with a flame ionisation detector and capillary Nukol column $(30 \mathrm{~m} \times 0.25 \mathrm{~mm}$; Supelco ${ }^{\circledR}$ ). $\mathrm{N}_{2}$ was used as the carrier gas and the flux in the column was $1.0 \mathrm{ml} / \mathrm{min}$. The temperatures of the injector and detector were set at 220 and $250^{\circ} \mathrm{C}$, respectively. Initial column temperature was $100^{\circ} \mathrm{C}$ sustained for $5 \mathrm{~min}$, rising at $10^{\circ} \mathrm{C} / \mathrm{min}$ until it reached $185^{\circ} \mathrm{C}$. Next, the samples were injected $(1 \mu \mathrm{l})$ through a Hamilton ${ }^{\circledR}$ syringe $(10 \mu \mathrm{l})$ in split system 5. The total run time was $33.5 \mathrm{~min}$. Concentrations were given as parts per million. In order to transform the results from parts per million to percentage of faecal mass, we considered that the concentration in parts per million represents the mass of the specific SCFA in $2 \mathrm{ml}$ of the solution, which in turn reflects the mass present in approximately $1600 \mathrm{mg}$ of faeces (the exact weight of faeces was used for calculation). The results were represented as per $100 \mathrm{mg}$ of faeces $(\% \mathrm{w} / \mathrm{w})$.

\section{Statistical analysis}

Statistical analyses were performed using the software Sigma Plot for Windows version 11.0 (Systat ${ }^{\circledR}$ Software). To assess 
Table 1. Clinical characteristics of the study subjects (Median and minimum (min)-maximum (max) values)

\begin{tabular}{|c|c|c|c|c|c|}
\hline \multirow[b]{2}{*}{ Variables } & \multicolumn{2}{|c|}{ Lean (n 20) } & \multicolumn{2}{|c|}{ Obese $(n 20)$} & \multirow[b]{2}{*}{$P$} \\
\hline & Median & Min-max & Median & Min-max & \\
\hline Systolic BP (mmHg) & 100 & $90-120$ & 120 & $90-130$ & $0.005^{\star}$ \\
\hline Diastolic BP (mmHg) & 60 & $50-80$ & 80 & $60-90$ & $0.002^{*}$ \\
\hline Weight $(\mathrm{kg})$ & $54 \cdot 6$ & $42 \cdot 2-64 \cdot 8$ & $88 \cdot 02$ & $74 \cdot 6-118 \cdot 1$ & $<0.001^{*}$ \\
\hline Height (cm) & $159 \cdot 5$ & $148 \cdot 5-173 \cdot 6$ & 159 & $150-168 \cdot 2$ & NS \\
\hline BMI $\left(k g / m^{2}\right)$ & $21 \cdot 2$ & $19 \cdot 22-23 \cdot 9$ & 34.4 & $29 \cdot 4-44 \cdot 6$ & $<0.001^{*}$ \\
\hline Waist (cm) & $68 \cdot 5$ & $65-77$ & $94 \cdot 5$ & $80 \cdot 5-118$ & $<0.001^{*}$ \\
\hline Body fat (\%) & $22 \cdot 6$ & $15 \cdot 8-28 \cdot 6$ & $37 \cdot 4$ & $29 \cdot 4-43$ & $<0.001 \dagger$ \\
\hline
\end{tabular}

if all the variables differed between the obese and lean groups, two different tests were used. Normally distributed variables were analysed by Student's $t$ test; otherwise, the Mann-Whitney test was used. Throughout the paper, the data are expressed as median (minimum-maximum). Spearman's correlation test was performed to measure the degree of correlation between each SCFA concentration and other metric variables. The level of significance was considered to be $5 \%$.

\section{Results}

\section{Subjects' characteristics}

As shown in Table 1, both obese and lean groups had normal blood pressure levels, but obese women presented higher medians of systolic and diastolic blood pressure $(P<0.05)$ compared to lean subjects. Weight, BMI, waist circumference and adiposity were significantly higher in the obese group $(P<0.05)$. All the obese volunteers showed waist circumference greater than $80 \mathrm{~cm}$. The biochemical variables are presented in Table 2 and it can be seen that subjects of both groups are normoglycaemic and normolipidaemic. However, fasting glucose was higher in the obese group $(P=0.027)$ as compared to lean subjects. The higher fasting insulin and
HOMA index $(P<0 \cdot 001)$ indicate the presence of insulin resistance in the obese group, which was detected in $45 \%$ of obese women. The obese group had a lower level of HDL compared to the lean group $(P=0.001)$. In addition, $90 \%$ of obese women ( $n$ 18) had HDL levels lower than $1.295 \mathrm{mmol} / \mathrm{l}$. The ratios of total cholesterol:HDL and LDL:HDL were statistically higher in the obese group compared to the lean group $(P<0 \cdot 05)$.

\section{Food intake}

The obese group presented a higher intake of energy, carbohydrates and lipids $(P<0.05)$ as compared to the lean group. The total intake of fibre was similar between both the groups assessed $(P=0.42)$. The median value of protein intake was slightly higher in the obese group $(P=0.058)$ than in the lean subjects (Table 3). No correlation was found between macronutrients and faecal SCFA concentration (data not shown).

\section{Faecal SCFA}

A higher percentage of butyric, acetic and propionic acids was observed in the faeces of the obese group $(P<0.05)$, as shown

Table 2. Biochemical characteristics of lean and obese women (Median and minimum (min)-maximum (max) values)

\begin{tabular}{|c|c|c|c|c|c|}
\hline & \multicolumn{2}{|c|}{ Lean (n 19) } & \multicolumn{2}{|c|}{ Obese $(n 20)$} & \multirow[b]{2}{*}{$P$} \\
\hline & Median & Min-max & Median & Min-max & \\
\hline $\mathrm{TC}(\mathrm{mmol} / \mathrm{l})$ & $4 \cdot 6$ & $3 \cdot 69-6 \cdot 6$ & $4 \cdot 29$ & $3 \cdot 1-5 \cdot 68$ & 0.33 \\
\hline $\mathrm{HDL}(\mathrm{mmol} / \mathrm{l})$ & $1 \cdot 34$ & $0.95-2.3$ & 1.08 & $0.77-1.96$ & $0.001^{*}$ \\
\hline $\mathrm{LDL}(\mathrm{mmol} / \mathrm{l})$ & $2 \cdot 62$ & $1.68-4.46$ & 2.60 & $1 \cdot 77-4 \cdot 35$ & 0.87 \\
\hline TAG (mmol/l) & 0.82 & $0.42-1.87$ & 0.95 & $0.41-1.62$ & 0.35 \\
\hline TC:HDL & 3.09 & $2 \cdot 23-6 \cdot 22$ & $3 \cdot 84$ & $2 \cdot 54-7 \cdot 33$ & $0.016^{*}$ \\
\hline LDL:HDL & 1.91 & $1.07-4.67$ & 2.51 & $1 \cdot 32-5 \cdot 61$ & $0.025^{\star}$ \\
\hline Fasting glucose $(\mathrm{mmol} / \mathrm{l})$ & $4 \cdot 77$ & $4 \cdot 16-5 \cdot 27$ & 4.96 & $4.6-5 \cdot 43$ & $0.027 \dagger$ \\
\hline Fasting insulin (pmol/l) & $56 \cdot 2$ & $34 \cdot 7-106 \cdot 2$ & $79 \cdot 1$ & $47 \cdot 2-252 \cdot 1$ & $<0.001^{*}$ \\
\hline HOMA & 1.65 & $0.96-3.47$ & 2.55 & $1 \cdot 39-8 \cdot 15$ & $<0.001^{*}$ \\
\hline AST (U/I) & 18 & $13-30$ & $17 \cdot 5$ & $14-28$ & 0.47 \\
\hline $\operatorname{ALT}(\mathrm{U} / \mathrm{l})$ & 13 & $7-30$ & 14 & $8-32$ & 0.82 \\
\hline
\end{tabular}


Table 3. Habitual macronutrient, fibre and energy intake of the lean and obese groups

(Median and minimum ( $\mathrm{min})$-maximum (max) values)

\begin{tabular}{|c|c|c|c|c|c|}
\hline & \multicolumn{2}{|c|}{ Lean $(n 18)$} & \multicolumn{2}{|c|}{ Obese $(n 19)$} & \multirow[b]{2}{*}{$P$} \\
\hline & Median & Min-max & Median & Min-max & \\
\hline Energy & & & & & $0.006^{*}$ \\
\hline kcal & $1669 \cdot 4$ & $1288 \cdot 7-2315 \cdot 5$ & $2206 \cdot 6$ & $1323.03-3309$ & \\
\hline $\mathrm{kJ}$ & $6984 \cdot 8$ & $5391 \cdot 9-9688 \cdot 1$ & 9232.4 & $5535 \cdot 6-13844.9$ & \\
\hline Carbohydrate $(\mathrm{g} / \mathrm{d})$ & $225 \cdot 8$ & $146 \cdot 4-310 \cdot 6$ & $277 \cdot 2$ & $143 \cdot 5-491 \cdot 6$ & $0.005 \dagger$ \\
\hline Proteins (g/d) & $57 \cdot 1$ & $41 \cdot 8-91 \cdot 8$ & 69.5 & $35 \cdot 3-123$ & $0.058^{*}$ \\
\hline Lipids (g/d) & 64.35 & $36 \cdot 7-91 \cdot 4$ & $78 \cdot 3$ & $51 \cdot 1-147$ & $0.01 \dagger$ \\
\hline Fibre $(g / d)$ & $16 \cdot 4$ & $11 \cdot 1-47.9$ & $13 \cdot 9$ & $8.0-55.5$ & $0.42^{*}$ \\
\hline
\end{tabular}

* Mann-Whitney.

†Student's $t$ test.

in Table 4. Also, a higher proportion of acetic acid was recorded in both groups. Additionally, the proportion of acetic:propionic:butyric acids, taking into account the median values, was also similar: $1 \cdot 4: 1: 1$ in the lean group and $1 \cdot 3: 1 \cdot 1: 1$ in the obese group.

Correlation tests were run for all variables analysed in the study, but only those which presented a statistically significant correlation $(P<0.05)$ were included in Table 5. Body fat, waist circumference, blood pressure, insulin and HOMA index were positively correlated with SCFA concentrations, while on the contrary, HDL was inversely correlated with butyric and acetic acids $(P<0 \cdot 05)$.

\section{Discussion}

Our present study is the first to investigate the association of faecal SCFA with clinical variables relevant to the diagnosis of the metabolic syndrome. The presence of these specific relationships suggests that SCFA modulation might be a target for weight management and abdominal obesity reduction, which are believed to improve metabolic risk factors and help in the prevention of metabolic diseases ${ }^{(17)}$.

In our study, we found that higher waist circumference was associated with an increase in SCFA concentration. Waist circumference has been considered a useful preliminary tool for the prediction of abdominal adiposity and metabolic syndrome screening ${ }^{(1,18)}$. The amount of intra-abdominal fat is related to a reduced insulin-stimulated glucose uptake rate in the skeletal muscle and in all fat depots ${ }^{(19)}$, suggesting that reduction of waist circumference is important for proper glucose metabolism and the loss of central fat depots.
We found that propionic and acetic acids positively correlated with waist circumference, weight, BMI and body fat percentage, demonstrating that SCFA might contribute to increased fat depots. Higher faecal concentrations of propionate and acetate induced by colonisation of germ-free mice with specific bacteria-producing SCFA resulted in higher weight and fat gain ${ }^{(20)}$. Acetate and especially propionate are signalling molecules for the GPR 41 receptor, the activation of which increases host adiposity ${ }^{(20)}$. The loss of GPR41 is associated with reduced efficiency to harvest energy from the diet. The interaction between the GPR41 receptors and SCFA results in increased absorption of SCFA used as a substrate for lipogenesis in the liver ${ }^{(20)}$. This shows that inhibition of SCFA activation of GPR41 could be a potential therapeutic target for control of fat gain. To reinforce this potential, acetate and propionate were also showed to inhibit lipolysis in cell culture, and thus favour lipid accumulation ${ }^{(21)}$.

Insulin levels and HOMA index are considered as markers of the metabolic syndrome related to fat accumulation ${ }^{(22)}$. In our study, a positive correlation of insulin levels and HOMA with the SCFA analysed was observed. The secretion of insulin can be activated by the stimulation of the receptor GPR40 in pancreatic cells. Long-chain NEFA can interact with GPR40 receptor, induce an increase in intracellular $\mathrm{Ca}^{2+}$ and amplify glucose-stimulated insulin secretion in a cell culture model. On the contrary, acetic and butyric acids do not induce insulin secretion $^{(23)}$. However, in overweight individuals the increase of acetate in blood, induced by lactulose intake, results in a slight increase in blood insulin, perhaps sufficient to decrease NEFA in plasma ${ }^{(24)}$ through the stimulation of lipoprotein lipase $^{(25)}$. Although this action helps to reduce plasma NEFA, it can favour higher body weight and fat percentage.

Table 4. Concentration ( $\% \mathrm{w} / \mathrm{w})$ of faecal SCFA from the lean and obese groups (Median and minimum ( $\mathrm{min})$-maximum ( $\max$ ) values)

\begin{tabular}{lllllll}
\hline & \multicolumn{2}{c}{ Lean group $(n$ 18) } & & \multicolumn{2}{c}{ Obese group $(n 17)$} & \\
\cline { 2 - 3 } SCFA $(\%)$ & Median & Min-max & & Median & Min-max & $P^{*}$ \\
\hline Butyric acid & 0.021 & $0.0038-0.095$ & & 0.044 & $0.009-0.285$ & 0.024 \\
Propionic acid & 0.0211 & $0.0088-0.055$ & & 0.0516 & $0.012-1.54$ & 0.007 \\
Acetic acid & 0.03 & $0.015-0.10$ & & 0.061 & $0.02-0.21$ & 0.010 \\
\hline * Mann-Whitney. & & & & &
\end{tabular}


Table 5. Correlation coefficient $(\rho)$ from comparison of faecal SCFA concentration with anthropometric, blood pressure and biochemical variables

\begin{tabular}{lccccrc}
\hline Variables & Butyric $(\rho)$ & \multicolumn{1}{c}{$P^{\star}$} & Propionic $(\rho)$ & $P^{\star}$ & Acetic $(\rho)$ & $P^{\star}$ \\
\hline Weight $(\mathrm{kg})$ & 0.34 & 0.049 & 0.44 & 0.008 & 0.46 & 0.006 \\
BMI $\left(\mathrm{kg} / \mathrm{m}^{2}\right)$ & 0.35 & 0.043 & 0.46 & 0.006 & 0.49 & 0.003 \\
Body fat $(\%)$ & 0.26 & 0.13 & 0.36 & 0.035 & 0.40 & 0.021 \\
Waist circumference (cm) & 0.37 & 0.034 & 0.43 & 0.011 & 0.50 & 0.002 \\
Systolic BP $(\mathrm{mmHg})$ & 0.40 & 0.020 & 0.38 & 0.027 & 0.44 & 0.010 \\
Diastolic BP (mmHg) & 0.32 & 0.065 & 0.34 & 0.047 & 0.35 & 0.042 \\
HDL (mmol/l) & -0.37 & 0.036 & -0.32 & 0.073 & -0.56 & 0.0008 \\
Insulin (pmol/l) & 0.46 & 0.007 & 0.53 & 0.001 & 0.58 & 0.0005 \\
HOMA index & 0.48 & 0.004 & 0.54 & 0.001 & 0.57 & 0.0005 \\
\hline
\end{tabular}

BP, blood pressure; HOMA, homeostasis model assessment.

* Spearman correlation test.

The role of SCFA in insulin secretion is thus controversial and needs further investigation.

In the present study, negative correlations were found between butyric and acetic acids and HDL. The influence of SCFA on HDL metabolism is not well established. Lower carbohydrate intake in a low-energy diet resulted in weight loss and an increase in HDL concentration ${ }^{(26)}$. On the other hand, a reduction in carbohydrate intake is associated with lower butyric acid production ${ }^{(27)}$. Based on these studies, one may assume a negative correlation between HDL and butyrate and acetate. However, the exact mechanism still needs to be discovered.

The quantity and proportion of different SCFA found in the gut reflect the amount and type of substrate in the diet (especially carbohydrates resistant to digestion, carbohydrates which escape absorption in the small intestine and also proteins), gut microbiota composition and activity, and transit time of the ingested food ${ }^{(10,28)}$. However, the specific mechanisms that could explain higher faecal SCFA concentrations in obese subjects are not yet clarified. Whether it is a result of an increased metabolic activity of specific bacterial groups, or a general increased intake of dietary substrates, still needs to be further investigated. The bacterial colonisation of the large intestine depends on the availability of dietary molecules that escape digestion and absorption by the host cells. The amount and type of such 'non-digestible' molecules, mainly carbohydrates, can influence the composition and metabolic activity of specific bacterial groups within the large-intestine environment ${ }^{(29)}$. The microbiome of obese individuals is enriched with several carbohydrate metabolic pathways ${ }^{(30)}$, which possibly could lead to increased production of SCFA. In our study population, higher dietary intake (energy, carbohydrates, lipids) was observed in obese women. In fact, higher dietary intake contributes to higher weight, waist circumference and body fat percentage. Thus, it is important to emphasise that although significant correlations were observed, our study design does not support a relationship of causality between SCFA and the clinical variables analysed. Higher SCFA could reflect an increased capacity of microbiota of obese subjects to harvest more energy from the diet, contributing directly to weight gain, adiposity and indirectly to insulin resistance. More studies are needed to assess if the higher concentrations of faecal SCFA are just a consequence of obesity or if they could directly contribute to the development of obesity and its metabolic complications.

Taken together, our results suggest that a higher faecal concentration of SCFA is associated with metabolic risk factors and thus may have an impact on waist circumference, adiposity, blood pressure, HOMA index, HDL and insulin levels. Thus, the modulation of faecal SCFA levels could be a target for metabolic homeostasis. However, the mechanisms explaining how SCFA can contribute to metabolic alterations need to be further investigated.

\section{Acknowledgements}

The present study was supported by FAPEMIG. T. F. S. T. is the recipient of a CAPES grant. T. F. S. T., J. B. and M. C. G. P. participated in the design and protocol of the study. T. F. S. T. carried out the study, S. C. C. F. helped with the statistical analyses and C. L. L. F. F. participated in the SCFA assays. T. F. S. T. and L. G. wrote the manuscript. None of the authors had a personal or financial conflict of interest.

\section{References}

1. Alberti KGMM, Eckel RH, Grundy SM, et al. (2009) Harmonizing the metabolic syndrome: a joint Interim Statement of the International Diabetes Federation Task Force on Epidemiology and Prevention; National Heart, Lung, and Blood Institute; American Heart Association; World Heart Federation; International Atherosclerosis Society; and International Association for the Study of Obesity. Circulation 120, $1640-1645$.

2. Simmons RK, Alberti KGMM, Gale EAM, et al. (2010) The metabolic syndrome: useful concept or clinical tool? Report of a WHO Expert Consultation. Diabetologia 53, 600-605.

3. Albala C, Vio F, Kain J, et al. (2002) Nutrition transition in Chile: determinants and consequences. Public Health Nutr 5, 123-128.

4. Després J-P \& Lemieux I (2006) Abdominal obesity and metabolic syndrome. Nature 444, 881-887.

5. Gastaldelli A, Natali A, Vettor R, et al. (2010) Insulin resistance, adipose depots and gut: interactions and pathological implications. Dig Liver Dis 42, 310-319. 
6. Bäckhed F, Ding H, Wang T, et al. (2004) The gut microbiota as an environmental factor that regulates fat storage. PNAS 101, 15718-15723.

7. Cani P, Neyrinck A, Fava F, et al. (2007) Selective increases of bifidobacteria in gut microflora improve high-fat-dietinduced diabetes in mice through a mechanism associated with endotoxaemia. Diabetologia 50, 2374-2383.

8. Blaut M \& Klaus S (2012) Intestinal microbiota and obesity. Handb Exp Pharmacol 209, 251-273.

9. Diamant M, Blaak EE \& de Vos WM (2011) Do nutrient-gutmicrobiota interactions plays a role in human obesity, insulin resistance and type 2 diabetes? Obes Rev 12, 272-281.

10. Wong JMW \& Jenkins DJA (2007) Carbohydrate digestibility and metabolic effects. J Nutr 137, 2539S-2546S.

11. Turnbaugh PJ, Ley RE, Mahowald MA, et al. (2006) An obesity-associated gut microbiome with increased capacity for energy harvest. Nature 444, 1027-1031.

12. Schwiertz A, Taras D, Schäfer K, et al. (2009) Microbiota and SCFA in lean and overweight healthy subjects. Obesity $\mathbf{1 8}$, $190-195$.

13. Friedewald WT, Levy RI \& Fredrickson DS (1972) Estimation of the concentration of low-density lipoprotein cholesterol in plasma, without use of the preparative ultracentrifuge. Clin Chem 18, 499-502.

14. Matthews DR, Hosker JP, Rudenski AS, et al. (1985) Homeostasis model assessment: insulin resistance and beta-cell function from fasting plasma glucose and insulin concentrations in man. Diabetologia 28, 412-419.

15. Geloneze B, Repetto EM, Geloneze SR, et al. (2006) The threshold value for insulin resistance (HOMA-IR) in an admixtured population. IR in the Brazilian Metabolic Syndrome Study. Diabetes Res Clin Prac 72, 219-220.

16. Smiricky-Tjardes MR, Grieshop CM, Flickinger EA, et al. (2003) Dietary galatooligasaccharides affect ileal and totaltract nutrient digestibility, ileal and fecal bacterial concentrations, and ileal fermentative characteristics of growing pigs. J Anim Sci 81, 2535-2545.

17. Wing RR, Lang W, Wadden TA, et al. (2011) Benefits of modest weight loss in improving cardiovascular risk factors in overweight and obese individuals with type 2 diabetes. Diabetes Care 34, 1481-1486.

18. Janssen I, Heymsfield SB, Allison DB, et al. (2002) Body mass index and waist circumference independently contribute to the prediction of nonabdominal, abdominal, subcutaneous, and visceral fat. Am J Clin Nutr 75, 683-688.

19. Virtanen KA, Iozzo P, Hällsten K, et al. (2005) Increased fat mass compensates for insulin resistance in abdominal obesity and type 2 diabetes. A Positron-Emitting Tomography Study. Diabetes 54, 2720-2726.

20. Samuel BS, Shaito A, Motoike T, et al. (2008) Effects of the gut microbiota on host adiposity are modulated by the short-chain fatty acid binding $G$ protein-coupled receptor, Gpr41. PNAS 105, 16767-16772.

21. Hong Y-H, Nishimura Y, Hishikawa D, et al. (2005) Acetate and propionate short chain fatty acids stimulate adipogenesis via GPCR43. Endocrinology 146, 5092-5099.

22. Arner P (2003) The adipocyte in insulin resistance: key molecules and the impact of the thiazolidinediones. TEM 14, $137-145$.

23. Itoh Y, Kawamata Y, Harada M, et al. (2003) Free fatty acids regulate insulin secretion from pancreatic $\beta$ cells through GPR40. Nature 422, 173-176.

24. Ferchaud-Roucher V, Pouteau E, Piloquet H, et al. (2005) Colonic fermentation from lactulose inhibits lipolysis in overweight subjects. Am J Physiol Endocrinol Metab 289, E716-E720.

25. Otarod J \& Goldberg IJ (2004) Lipoprotein lipase and its role in regulation of plasma lipoproteins and cardiac risk. Curr Atheroscler Rep 6, 335-342.

26. Sacks FM, Bray GA, Carey VJ, et al. (2009) Comparison of weight-loss diets with different compositions of fat, protein, and carbohydrates. $N$ Engl J Med 360, 859-873.

27. Duncan SH, Belenguer A, Holtrop G, et al. (2007) Reduced dietary intake of carbohydrates by obese subjects results in decreased concentrations of butyrate and butyrateproducing bacteria in feces. Appl Environ Microbiol $\mathbf{7 3}$ 1073-1078.

28. Macfarlane S \& Macfarlane GT (2003) Regulation of shortchain fatty acid production. Proc Nutr Soc 62, 67-72.

29. Flint HJ, Bayer EA, Rincon MT, et al. (2008) Polysaccharide utilization by gut bacteria: potential for new insights from genomic analysis. Nature 6, 121-131.

30. Turnbaugh P, Hamady M, Yatsunenko T, et al. (2009) A core gut microbiome in obese and lean twins. Nature $\mathbf{4 5 7}$, 480-484. 